Check for updates

Cite this: RSC Adv., 2017, 7, 40745

Received 20th July 2017 Accepted 12th August 2017

DOI: $10.1039 / c 7 r a 08007 g$

rsc.li/rsc-advances

\section{Effect of soft segment molecular weight on the glass transition, crystallinity, molecular mobility and segmental dynamics of poly(ethylene oxide) based poly(urethane-urea) copolymers}

\author{
Oguzhan Oguz, (DD ${ }^{\text {ab }}$ Stefanos A. Koutsoumpis, ${ }^{c}$ Eren Simsek, $\dagger^{\text {ab }}$ Emel Yilgor, ${ }^{d}$ \\ Iskender Yilgor, ${ }^{* d}$ Polycarpos Pissis*c and Yusuf Z. Menceloglu (DD *ab
}

\begin{abstract}
The effect of poly(ethylene oxide) (PEO) soft segment molecular weight $\left(M_{n}=2000,4600\right.$ and $8000 \mathrm{~g}$ $\mathrm{mol}^{-1}$ ) on the glass transition, crystallinity, molecular mobility and segmental dynamics of a series of aliphatic polyurethaneurea copolymers (PUU) with a constant hard segment content of $30 \%$ by weight was investigated using differential scanning calorimetry and dielectric relaxation spectroscopy. The soft segment (PEO) glass transition temperature increased with increasing molecular weight. Furthermore, five different relaxations were observed in dielectric analyses of all copolymers. These included local glassy state motions $(\gamma)$ and $(\beta)$, segmental motion of the soft phase $(\alpha)$, conductivity relaxation, and interfacial Maxwell-Wagner-Sillars (MWS) polarization. Local relaxations follow Arrhenius behavior and their time scale is not affected by the soft segment molecular weight. $\alpha$-Relaxation follows VogelTammann-Fulcher (VTF) behavior and is slower for the copolymer based on PEO-4600. Conductivity relaxation and the interfacial MWS polarization also follow VTF behavior and have quite similar slopes since both are related to the same phenomena. Unexpectedly, the interfacial MWS polarization is not affected by the soft segment molecular weight. Although this result suggests that the soft segment molecular weight does not affect the microphase separation in these copolymers, we believe that no safe conclusions can be extracted for this system due to the high complexity and the presence of many phases with different conductivity. However, significant differences were observed in the conductivity relaxation, which is much faster for the copolymer based on PEO-2000, due to its lower crystallinity when compared with others.
\end{abstract}

\section{Introduction}

Segmented polyurethaneurea copolymers (PUU) consist of alternating soft and hard segments chemically linked together along a linear macromolecular backbone. Due to their versatile chemistry and interesting combination of properties, PUUs can be utilized in a wide range of applications, such as coatings, biomaterials and textiles fibers. ${ }^{\mathbf{1 - 4}}$ In general, soft segments (SS) are hydroxyl or amine terminated aliphatic polyethers,

\footnotetext{
${ }^{a}$ Faculty of Engineering and Natural Sciences, Materials Science and Nano Engineering, Sabanci University, 34956, Orhanli, Tuzla, Istanbul, Turkey. E-mail: yusufm@sabanciuniv.edu

${ }^{b}$ Sabanci University Integrated Manufacturing Technologies Research and Application Center, Composite Technologies Center of Excellence, Teknopark Istanbul, 34906 Pendik, Istanbul, Turkey

${ }^{c}$ Department of Physics, National Technical University of Athens, Iroon Polytechneiou 9, Zografou Campus, 157 80, Athens, Greece. E-mail: ppissis@central.ntua.gr ${ }^{d}$ KUYTAM Surface Science and Technology Center, Chemistry Department, Koc University, Sariyer, 34450, Istanbul, Turkey. E-mail: iyilgor@ku.edu.tr

$\dagger$ Currently at Quantag Nanotechnologies, Urla, Izmir, Turkey.
}

polyesters, polycarbonates, polyisobutylene and polydimethylsiloxane oligomers with glass transition temperatures $\left(T_{\mathrm{g}}\right)$ well below room temperature. Hard segments (HS) consist of strongly hydrogen bonded urethane and/or urea groups, which are obtained by the reaction of diisocyanates with diol or diamine chain extenders. They mainly serve as stiff, physically cross-linked domains with a $T_{\mathrm{g}}$ or $T_{\mathrm{m}}$ well above the service temperature range. Availability of a large selection of starting materials provides opportunities for the preparation of a wide range of PUU backbone structures with different properties. PUUs display composition dependent microphase morphologies or nanostructures and interesting combination of bulk and surface properties. ${ }^{1-4}$ However, microphase separation, crystallization, molecular mobility and segmental dynamics are quite complex due to the presence of large number of parameters which need to be controlled during PUU synthesis, processing and thermal annealing. These parameters mainly include chemical structure, size and distribution of sequence lengths and HS/SS ratio, which leads to various equilibrium morphologies ranging from mixed amorphous SS/HS phases to 
well microphase separated morphologies. Therefore, a good understanding of the relationships between microphase separation, segment crystallization, molecular mobility and segmental dynamics is of particular interest to tailor the final properties of PUUs.

Fairly large number of studies ${ }^{3,4}$ have been, and continues to be, carried out on the investigation of the structuremorphology-property behavior of segmented PUUs. Interestingly, number of systematic studies focusing on the molecular mobility and segmental dynamics in PUUs are considerably limited in the literature..$^{5-9}$ Furthermore, segmented copolymers with a relatively narrow range of soft segment molecular weights (generally, up to $2000 \mathrm{~g} \mathrm{~mol}^{-1}$ ) and urethane type (rather than urea) hard segments were utilized in most of these studies. More specifically, although widely used in the design and synthesis of segmented PUUs, to the best of our knowledge, the molecular mobility and segmental dynamics of poly(ethylene oxide) (PEO) based PUUs have not been investigated in detail as a function of soft segment molecular weight. From this perspective, here, we mainly focused on the molecular mobility and segmental dynamics of a series of PEO-based segmented PUU copolymers with an identical hard segment content of $30 \mathrm{wt} \%$, using differential scanning calorimetry (DSC) and dielectric relaxation spectroscopy (DRS). DSC is a well-known technique to investigate the thermal properties of polymeric materials. ${ }^{\mathbf{1 0 , 1 1}}$

On the other hand, DRS ${ }^{\mathbf{1 2}}$ is a powerful technique to investigate the dynamics of complex polymeric systems over a very broad range of frequencies. Thus, morphology dependent dynamic processes taking place in polymers, which are on multiple length scales and different time scales can be identified in an effort to understand the structural states of these materials. ${ }^{13-28}$ To date, various polymeric materials have been, and continues to be, analyzed by DRS. ${ }^{13-20}$ In polyurethanes, this technique is mainly used to investigate the local dynamics, dynamic glass transition and indirectly, crystallinity and microphase morphology. ${ }^{21-28}$ Combination of DSC and DRS enables us to gain a complete understanding of the structural variables, such as soft segment type and molecular weight and hard segment structure and content on the molecular mobility and segmental dynamics in polyurethanes.

PEO is a convenient choice for the design of the soft segments in PUUs due to the following reasons; (i) availability of reactive PEO oligomers with a wide range of molecular weights, (ii) conformational properties of the ethylene oxide units and molecular weight dependent crystallization of PEO blocks leading to various physical states in PUUs, (iii) amphiphilic nature of PEO chains due to the presence of hydrophobic methylene groups and hydrophilic oxygen atoms that can participate in hydrogen bonding, and (iv) presence of extensive literature data on the folding and crystallization behavior of PEOs as a function of chain length. ${ }^{29-35}$ Therefore, the use of PEOs with various molecular weights allows for the preparation of PUUs with different morphologies. However, PEO based PUUs also present some challenges due to their structurally and dynamically heterogeneous building blocks. One of these challenges is the investigation of the molecular mobility and segmental dynamics of these copolymers due to high conductivity of PEO. In this study, we focus on the effect of PEO molecular weight on the glass transition, crystallinity, molecular mobility and segmental dynamics in PEO based PUU copolymers.

\section{Experimental}

\subsection{Materials}

Poly(ethylene oxide)glycol oligomers (PEO) with $\left\langle M_{\mathrm{n}}\right\rangle$ values of 2000, 4600 and $8000 \mathrm{~g} \mathrm{~mol}^{-1}$ were purchased from Merck. Bis(4-isocyanatocyclohexyl)methane (HMDI) was provided by Bayer and had a purity better than 99.5\%. Chain extender, 2-methyl-1,5-diaminopentane (MDAP) was supplied by DuPont. Dibutyltin dilaurate (DBTDL) was obtained from Witco and was used as catalyst by diluting to $1 \%$ by weight in tetrahydrofuran. Reagent grade 2-propanol (IPA), tetrahydrofuran (THF) and dimethylformamide (DMF) were obtained from Merck. All chemicals were used as received.

\subsection{Synthesis of segmented poly(ether-urethane-urea) copolymers}

PUUs were prepared by prepolymer method outlined in our previous studies., ${ }^{\mathbf{4} 36}$ Reactions were carried out in three-neck, round bottom, Pyrex reaction flasks equipped with an overhead stirrer, thermometer and an addition funnel. Isocyanate terminated prepolymer was prepared in THF $(50 \%$ solids by weight) at $60{ }^{\circ} \mathrm{C}$ by reacting PEO and HMDI, under the catalysis of $150 \mathrm{ppm}$ of DBTDL. Progress and completion of the prepolymer reaction was monitored by FTIR spectroscopy. After the completion of prepolymer reaction in about $1 \mathrm{~h}$, the solution was cooled down to room temperature and diluted to $25 \%$ by weight of solids using DMF. MDAP was dissolved in DMF (about $10 \%$ by weight) and added dropwise from the addition funnel into the reaction mixture until the complete disappearance of the isocyanate peak at $2260 \mathrm{~cm}^{-1}$. The viscosity increase during the chain extension reaction was controlled by adding DMF into the reaction flask. Chemical structure of the PUU copolymers is shown in Fig. 1.

\subsection{Preparation of polymer films}

Polymer films were prepared by solution casting in a Teflon mold. Solvent was first evaporated at room temperature overnight in a fume hood, followed by drying in an air oven at $60{ }^{\circ} \mathrm{C}$ for 24 hours. Complete removal of the solvents was achieved by drying the films in a vacuum oven at $60{ }^{\circ} \mathrm{C}$ until constant weight. This was also confirmed by thermogravimetric analyses of the film samples (data not shown in the study). Samples based on PEO-2000, 4600 and 8000 with 30 wt\% urethane-urea HS were denoted as PU-2, PU-5 and PU-8, respectively. After annealing at $60{ }^{\circ} \mathrm{C}$ for 24 hours the films were kept at room temperature in a desiccator for a week, to achieve equilibrium morphologies.

\subsection{Characterization techniques}

Gel Permeation Chromatography (GPC) analyses were performed using a Viscotek GPCmax VE-2001 instrument equipped 


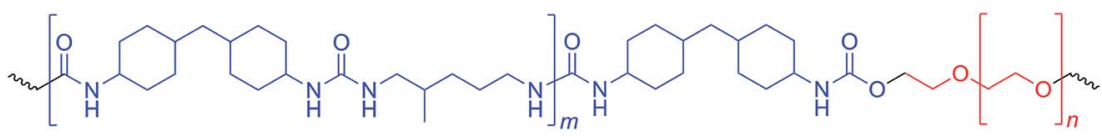

Fig. 1 Chemical structure of PUUs based on PEO, HMDI and MDAP.

with D 5000-D 3000-D 1000-D Guard columns and RI, LS, DP detectors. DMF was used as the solvent and analysis was performed at $55{ }^{\circ} \mathrm{C}$ with a flow rate of $1 \mathrm{~mL} \mathrm{~min}^{-1}$. Polymer solutions were prepared in DMF at a concentration of $2 \mathrm{mg} \mathrm{mL}^{-1}$. The samples were filtered using VMR PTFE syringe filters with average pore size of $0.45 \mu \mathrm{m}$ before measurements. Average molecular weights were determined using calibration curves obtained from polystyrene standards. PU-2, PU-5 and PU-8 have weight average molecular weights of 100000,232000 and $309000 \mathrm{~g} \mathrm{~mol}^{-1}$, and number average molecular weights of 68000,164000 and $209500 \mathrm{~g} \mathrm{~mol}^{-1}$ with molecular weight distributions of 1.48, 1.42 and 1.47 , respectively.

X-ray diffraction (XRD) studies of the film samples (an average thickness of $0.2 \mathrm{~mm}$ ) were performed on a Bruker D8 Advance X-ray Diffractometer using Ni-filtered $\mathrm{Cu}-\mathrm{K} \alpha$ radiation $(\lambda=0.1542 \mathrm{~nm})$. All measurements were performed from 5 up to $90^{\circ}$ with a step size of $0.01^{\circ}$. The results were plotted as a function of the scattering angle $2 \theta$ without applying any fitting procedure.

Stress-strain analyses were performed on dog-bone type specimens punched out of thin films using a standard die (ASTM D 1708). Measurements were made on a Zwick Z100 model tester under ambient temperature and humidity conditions with a crosshead speed of $25.0 \mathrm{~mm} \mathrm{~min}^{-1}\left(L_{\mathrm{o}}=24.0 \mathrm{~mm}\right)$. At least five specimens were tested for each material.

Differential Scanning Calorimetry (DSC) analyses were performed on a TA Q2000 instrument calibrated with indium standard and equipped with $T_{\text {zero }}$ functionality that significantly improves the baseline via compensating resistance and capacitance imbalances. All measurements were performed in the range of -160 and $250{ }^{\circ} \mathrm{C}$ at a heating and cooling rate of $3{ }^{\circ} \mathrm{C} \min ^{-1}$ under nitrogen atmosphere. Glass transition temperature $\left(T_{\mathrm{g}}\right)$ and the heat capacity change at $T_{\mathrm{g}}\left(\Delta C_{\mathrm{p}}\right.$ at $\left.T_{\mathrm{g}}\right)$ were determined by the inflection point and the tangent methods, respectively. The degree of crystallinity (crystalline fraction $-X_{\mathrm{c}}$ ) is calculated using the melting enthalpy normalized by the weight fraction of the hard segment, assuming that it does not contribute to the crystallinity of the soft segment:

$$
X_{\mathrm{c}}=\frac{\Delta H_{\mathrm{m}}}{\left(1-X_{\mathrm{HS}}\right) \times \Delta H_{100 \%, \mathrm{PEO}}} \times 100 \%
$$

where $\Delta H_{\mathrm{m}}$ is the melting enthalpy obtained from the area of the melting peak, $X_{\mathrm{HS}}$ is the weight fractions of the hard segment. The melting enthalpy for the $100 \%$ crystalline polymer is considered to be equal to that of pure PEO $\left(\Delta H_{100 \%, \mathrm{PEO}}\right)$, which is denoted as $196.8 \mathrm{~J} \mathrm{~g}^{-1} \cdot{ }^{34}$

Dielectric Relaxation Spectroscopy (DRS) studies were conducted on a Novocontrol Alpha Analyzer. Samples were placed between two brass electrodes, forming a capacitor of known area and thickness equal to the thickness of each sample. The complex dielectric function $\varepsilon^{*}$ was studied in the frequency range of $10^{-1}$ to $10^{6} \mathrm{~Hz}$. Temperature was varied from $-150{ }^{\circ} \mathrm{C}$ to $20{ }^{\circ} \mathrm{C}$ for PU-2 and PU-5 and from $-60{ }^{\circ} \mathrm{C}$ to $20^{\circ} \mathrm{C}$ for PU-8. The relaxations are evident as peaks in the dielectric loss $\varepsilon^{\prime \prime}$ spectra, which can be described using the Havriliak-Negami $(\mathrm{H}-\mathrm{N})$ function: ${ }^{37}$

$$
\varepsilon^{\prime \prime}(f)=\operatorname{Im}\left[\frac{\Delta \varepsilon}{\left[1+\left(\text { if } / f_{\mathrm{HN}}\right)^{a}\right]^{b}}\right]
$$

where $\left(f_{\mathrm{HN}}\right)$ is a characteristic frequency related to the frequency of maximum loss $\left(f_{\max }\right),(\Delta \varepsilon)$ is the relaxation strength and $(a)$ and $(b)$ are shape parameters. Temperature dependence of the time scale of the dielectric response can be followed through the Arrhenius plot (plot of the logarithm of the frequency of the dielectric loss peak against reciprocal temperature) and can be further analyzed by fitting appropriate equations. The Arrhenius equation is common for describing local dynamics, ${ }^{38}$

$$
f_{\text {max }}(T)=f_{0} \exp \left(-\frac{E_{\text {act }}}{k T}\right)
$$

where $\left(f_{\max }\right)$ is the frequency of $\varepsilon^{\prime \prime}(f)$ peak, $(T)$ the temperature, $\left(f_{0}\right)$ a pre-exponential constant, $E_{\text {act }}$ the activation energy of the relaxation and $(k)$ the Boltzmann's constant. The Vogel-Tammann-Fulcher (VTF) equation, characteristics of cooperative processes, was used to describe the time scale dependence on temperature, ${ }^{38}$

$$
f_{\max }(T)=f_{0} \exp \left(-\frac{B}{T-T_{0}}\right)
$$

where $(B)$ is the apparent activation energy, $\left(f_{0}\right)$ the preexponential frequency factor and $\left(T_{0}\right)$ the Vogel temperature. The strength parameter was calculated as $D=B / T_{0}$ and the fragility as $m=16+590 / D .^{39}$

Another formalism we can use is that the electric modulus $(M)$, defined as $M^{*}=1 / \varepsilon^{*}$, which has been proven effective in analyzing ionic conductivity and interfacial effects in amorphous materials. ${ }^{40}$ Conductivity and conductivity effects were analyzed in terms of ac conductivity $\left(\sigma_{\mathrm{ac}}\right)$ calculated as $\sigma_{\mathrm{ac}}=$ $2 \pi f \varepsilon_{0} \varepsilon^{\prime \prime}(f)$, where $\left(\varepsilon_{0}\right)$ is the permittivity of free space. ${ }^{\mathbf{4 0}}$

\section{Results and discussion}

To provide a clear overview on the dynamics of PU copolymers, it is important to know the structural characteristics of PU copolymers along with their microphase morphologies and final properties. For this reason, before going into the results and discussion of dielectric studies, we report the results obtained from XRD, DSC and stress-strain analyses of the samples under investigation. Fig. 2 shows the room temperature XRD patterns of PUU samples. The XRD results basically suggest that 
PEO matrix in PU-2 is mainly amorphous, while those in PU-5 and PU-8 are semi-crystalline at room temperature.

As shown in Fig. 2, PU-5 and PU-8 display typical X-ray diffraction patterns widely observed for monoclinic crystal structure of helical PEO chains, ${ }^{29,31,34}$ whereas PU-2 shows an amorphous halo. The scattering angles of the main XRD peaks observed in PU-5 and PU-8 for crystalline PEO segments are in good agreement with those reported in the literature for the helical model of the crystalline PEO along with their $h k l$ indexing and lattice parameters of the monoclinic unit cell listed as (a): $0.805 \mathrm{~nm},(\mathrm{~b}): 1.304 \mathrm{~nm}$ and (c): $1.948 \mathrm{~nm}$ with $\beta=$ $125.4^{\circ}$ (the oblique angle between the $a$ - and $c$-axis)..$^{28,30,33}$ The (120) reflections $\left(2 \theta=19.3^{\circ}\right)$ taken along the [001] zone axis have information about interchain separations and chain conformations, i.e., the orientation of the helix, whereas the (112) reflections $\left(2 \theta=23.2^{\circ}\right)$ contains structural information along the $c$-axis, i.e., the helicity of PEO macromolecules. ${ }^{41}$ This may be a convenient framework to investigate the presence of a "secondary structure" in crystalline parts of soft domains in PU-5 and PU-8 in a further study.

Thermal properties of PUs are strongly dependent on the annealing conditions. In this study, all samples were annealed for 24 hours at $60{ }^{\circ} \mathrm{C}$ and then kept at room temperature in a desiccator for a week, to achieve equilibrium morphologies. DSC thermograms of PU copolymers are provided in Fig. 3. The results obtained from the thermal analyses of the samples are listed in Table 1. As can be seen in Fig. 3 and Table 1, PU-2 copolymer shows a well-defined glass transition at $-57.2{ }^{\circ} \mathrm{C}$ with a heat capacity change $\left(\Delta C_{\mathrm{p}}\right.$ at $\left.T_{\mathrm{g}}\right)$ of $0.485 \mathrm{~J} \mathrm{~g}^{-1} \mathrm{~K}^{-1}$, followed by a small exotherm between -50 and $-25{ }^{\circ} \mathrm{C}$ and an endotherm at $19.9{ }^{\circ} \mathrm{C}$. PU-5 and PU-8 copolymers, which are highly crystalline when compared to PU-2, display very weak glass transition temperatures at -51.9 and $-48.9^{\circ} \mathrm{C}$ with $\Delta C_{\mathrm{p}}$ at $T_{\mathrm{g}}$ values of 0.113 and $0.058 \mathrm{~J} \mathrm{~g}^{-1} \mathrm{~K}^{-1}$, followed by sharp endotherms at 42.1 and $51.2{ }^{\circ} \mathrm{C}$, respectively. The glass transitions at $-57.2,-51.9$ and $-48.9{ }^{\circ} \mathrm{C}$ are assigned to the PEO soft segments of PU-2, PU-5 and PU-8 copolymers, respectively. As expected, $T_{\mathrm{g}}$ of PEO soft segment increases with increasing molecular weight. ${ }^{42}$ As listed in Table 1 , the endothermic peaks at 19.9, 42.1 and $51.2{ }^{\circ} \mathrm{C}$ are attributed to the melting points $\left(T_{\mathrm{m}}\right)$ of PEO soft segment crystals in PU-2, PU-5 and PU-8,

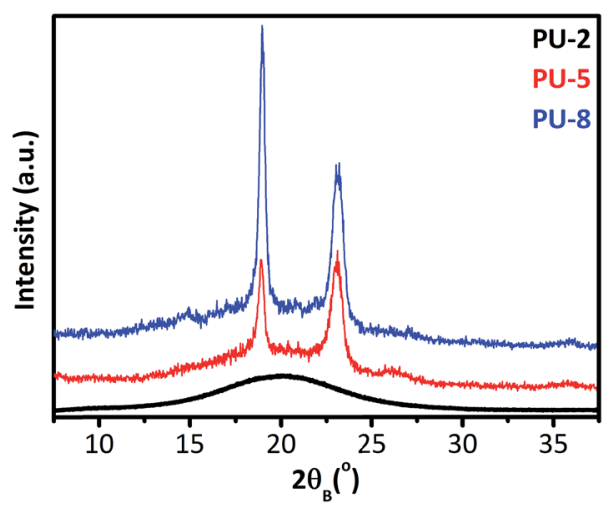

Fig. 2 XRD patterns of PU-2, PU-5 and PU-8. respectively. The degree of soft segment crystallinity $\left(X_{\mathrm{c}}\right)$ values are calculated as $32.6,43.5$ and $56.4 \%$ for PU-2, PU-5 and PU-8, respectively. $T_{\mathrm{m}}$ and $X_{\mathrm{c}}$ values also increases with increasing molecular weight. No other high temperature exothermic and/or endothermic transitions are observed for any samples. As listed in Table 1, a decreasing trend in the $\Delta C_{\mathrm{p}}$ at $T_{\mathrm{g}}$ is observed. In line with the increasing trend in $T_{\mathrm{g}}$, the decreasing trend in $\Delta C_{\mathrm{p}}$ at $T_{\mathrm{g}}$ indicates the restriction imposed on the chain movements due to increasing soft segment crystallinity as a direct result of increasing soft segment molecular weight. It should be also noted that the magnitude of $\Delta C_{\mathrm{p}}$ at $T_{\mathrm{g}}$ is significantly higher in PU-2 copolymer when compared with those of PU-5 and PU-8. This is due to that PEO soft segments in PU-5 and PU-8 are inherently more crystalline when compared with PU-2. These results are in accordance with the results obtained from XRD analyses of the copolymers as given in Fig. 2. To clarify, one should note that the melting endotherm observed in PU-2 copolymer is below room temperature, and thus, X-ray diffraction pattern shows an amorphous halo. In contrast, the melting endotherms observed in PU-5 and PU-8 are far above room temperature. Therefore, their XRD patterns clearly display the characteristic peaks of PEO crystals as described earlier.

In addition to the results obtained from XRD and DSC analyses, the stress-strain properties of the copolymers are also provided in Table 2. As listed in Table 2, the tensile test results clearly demonstrate the formation of high strength elastomers, with ultimate tensile strength values around $30 \mathrm{MPa}$, under the applied processing condition. Since the HS content is constant for all the copolymers reported in here, no significant difference is observed in the ultimate tensile strengths. The elastic modulus values of the copolymers increase with increasing PEO molecular weight. In line with $\mathrm{XRD}$ and DSC results, the modulus of PU-5 and PU-8 with highly crystalline PEO matrices are significantly higher than the one of PU-2 with an amorphous PEO matrix at room temperature. Accordingly, the elongation at break value of PU-2 is remarkably higher than the ones of PU-5 and PU-8 copolymers due to the presence of highly mobile amorphous matrix phase in this copolymer. In addition, the elongation at break values decrease with increasing PEO molecular weight.

\subsection{Dielectric relaxation spectroscopy (DRS) studies}

Five different relaxations were observed in dielectric analyses of all copolymers: local glassy state motions $(\gamma$ and $\beta)$, segmental motion of the soft phase $(\alpha)$, conductivity relaxation (CR), and interfacial Maxwell-Wagner-Sillars (MWS) polarization. All of them are discussed in detail in the following sections along with the room temperature measurements.

3.1.1. Glassy state motions. In the glassy state, PU-2, PU-5 and PU-8 have two local molecular motions: $\beta$-relaxation (at low frequencies) and $\gamma$-relaxation (at high frequencies). The $\gamma$ peak has been associated particularly with the crankshaft motions of the ether oxygen containing segments.5,7,26 The $\beta$-relaxation is known to be related to the reorientational motions of water molecules and/or local motions of polymer 

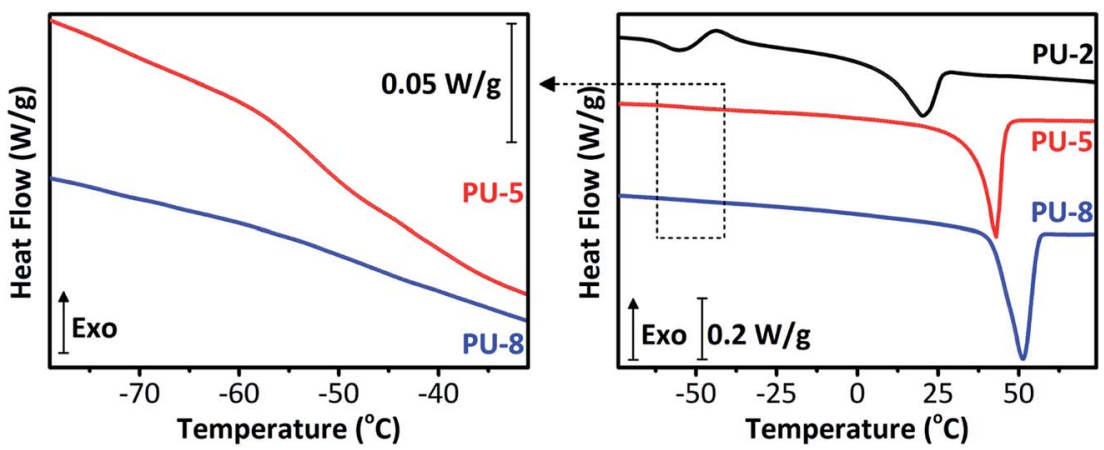

Fig. 3 The glass transition region in DSC thermograms of PU-5 and PU-8 (left) and complete DSC thermograms of PU-2, PU-5 and PU-8 (right) in the range of -75 and $75^{\circ} \mathrm{C}$.

chain segments attached to the water molecules, which is widely observed in hydrated materials and/or polyurethanes reported in the literature..$^{5,7,27,28}$ This is mainly because of the strong interaction between the polar hard segments and ether linkages of the PEO segments and some residual water present in these materials containing highly hydrophilic PEO matrices, even though they were well dried prior to analyses. The dielectric losses at two selected temperatures $\left(-80\right.$ and $\left.-60{ }^{\circ} \mathrm{C}\right)$ are representatively shown in Fig. 4 for all three samples.

As can be seen in Fig. 4 , two peaks are observed at $-80^{\circ} \mathrm{C}$. The peak at low frequencies corresponds to the $\beta$-relaxation, whereas the one at high frequencies is attributed to the $\gamma$-relaxation. As expected, these two peaks are shifted to the higher frequencies by increasing the temperature due to the thermally activated polarization phenomena. This can also be clearly seen in the spectra recorded at $-60{ }^{\circ} \mathrm{C}$ in Fig. 4. Moreover, the frequency of the peaks is the same for all three samples. Thus, the relaxation times of the $\gamma$ and $\beta$ relaxations are not significantly affected by PEO molecular weight. It should also be noted that the relative heights of these two peaks are different for PU-5 compared to the other two samples.

To quantify the results, Arrhenius plots are provided for these two relaxations in Fig. 5. For each isotherm, one Havriliak-Negami function was fitted to $\beta$ and $\gamma$ relaxations. Temperature-frequency pairs of the relaxation peaks were determined when they started to converge during the fitting process. ${ }^{37}$ It should be noted that PU-8 was not systematically measured at low temperatures compared to PU-2 and PU-5 since the local relaxations ( $\beta$ and $\gamma$ ) were at the same frequency for all three samples as shown in Fig. 4. For this reason, the Arrhenius plot for $\beta$ and $\gamma$ relaxations provided in Fig. 5 is constructed using the data obtained from the analyses of PU-2 and PU-5. As expected, both of the relaxations ( $\beta$ and $\gamma$ ) follow a straight line, which is characteristic for local motions. ${ }^{38}$
Table 2 Tensile properties of PU-2, PU-5 and PU-8

\begin{tabular}{lccc}
\hline & & \multicolumn{2}{l}{ Tensile strength } \\
Sample code & Modulus $(\mathrm{MPa})$ & Elongation (\%) \\
\hline PU-2 & $5.0 \pm 0.5$ & $30 \pm 1$ & $1000 \pm 40$ \\
PU-5 & $140 \pm 14$ & $29 \pm 1$ & $620 \pm 20$ \\
PU-8 & $360 \pm 15$ & $27 \pm 2$ & $430 \pm 30$
\end{tabular}

Activation energies calculated for these two relaxations from Fig. 5 are listed in Table 3. The activation energy for $\beta$-relaxation $\left(0.67 \mathrm{eV} \approx 64.7 \mathrm{~kJ} \mathrm{~mol}^{-1}\right)$ is almost double of that of $\gamma$-relaxation $\left(0.38 \mathrm{eV} \approx 36.7 \mathrm{~kJ} \mathrm{~mol}^{-1}\right)$, even though these two relaxations have similar time scales. This is counterbalanced by the high value of $\left(f_{0}\right)$ for the $\beta$-relaxation. The parameter $\left(f_{0}\right)$ is related with lattice vibrations. The unexpectedly high value of $f_{0}$ reflects some cooperativity in this relaxation..$^{38}$ In general, the activation parameters of the $\beta$-relaxation show a scattering in the literature, ${ }^{26,28,43}$ most probably reflecting different hydration levels of the samples studied.

3.1.2. Segmental relaxation. Aforementioned, PEO SS glass transition temperatures for PU-2, PU-5 and PU-8, respectively, were recorded as $-57.8,-51.9$ and $-48.4{ }^{\circ} \mathrm{C}$ by DSC analyses. Thus, during the dielectric measurements, one should expect to observe the $\alpha$-relaxation or the dynamic glass transition around the glass transition temperatures obtained from the calorimetry. ${ }^{24}$ However, a jump in the magnitude of $\varepsilon^{\prime \prime}$ values, which is mainly due to the overall increase of the conductivity regarding to increasing temperature, was observed around the calorimetric glass transition temperatures, the peak of $\alpha$-relaxation was not entered into the frequency window. This can be clearly seen from the different isotherms given in Fig. 6.

The isotherms given in Fig. 6 clearly indicate that a more complex analysis is needed for a better understanding of the

Table 1 Thermal properties of PU-2, PU-5 and PU-8

\begin{tabular}{lllllr}
\hline Sample & $T_{\mathrm{g}}\left({ }^{\circ} \mathrm{C}\right)$ & $\Delta C_{\mathrm{p}}$ at $T_{\mathrm{g}}\left(\mathrm{J} \mathrm{g}^{-1} \mathrm{~K}^{-1}\right)$ & $T_{\mathrm{m}}\left({ }^{\circ} \mathrm{C}\right)$ & ${ }^{\mathrm{DSC}} \Delta H_{\mathrm{m}}\left(\mathrm{J} \mathrm{g}^{-1}\right)$ & ${ }^{\text {normalized }} \Delta H_{\mathrm{m}}\left(\mathrm{J} \mathrm{g}^{-1}\right)$ \\
\hline PU-2 & -57.2 & 0.485 & 19.9 & 44.9 & 64.1 \\
PU-5 & -51.9 & 0.113 & 42.1 & 59.9 & 85.5 \\
PU-8 & -48.9 & 0.058 & 51.2 & 77.6 & 110.9
\end{tabular}




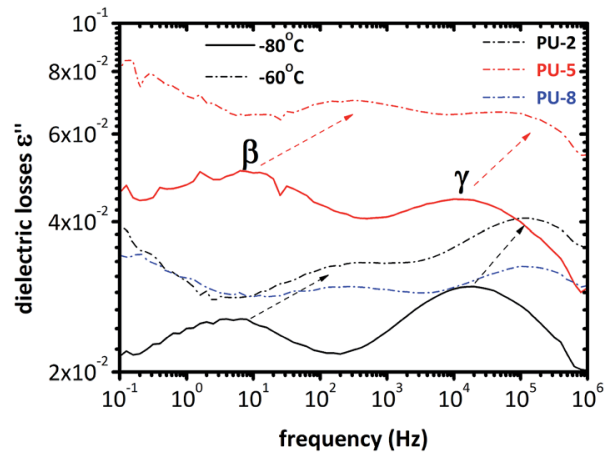

Fig. 4 Dielectric losses at $-80{ }^{\circ} \mathrm{C}$ and $-60{ }^{\circ} \mathrm{C}$ for PU-2, PU-5 and $\mathrm{PU}-8$. Two local relaxations, $\beta$ and $\gamma$, are within the frequency windows in these temperatures.

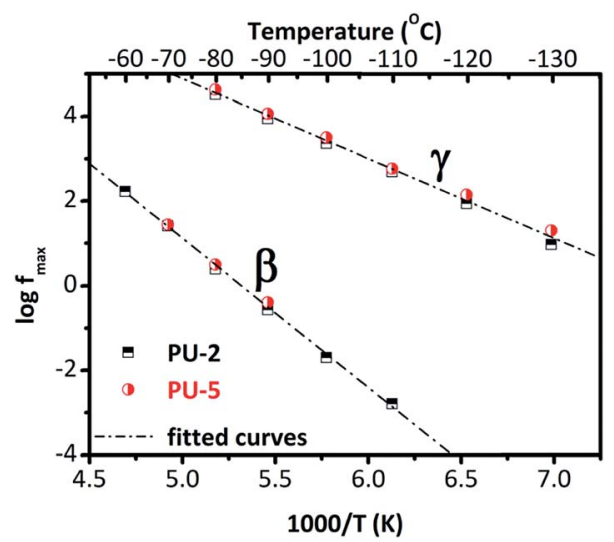

Fig. 5 Arrhenius plot for the $\beta$ and $\gamma$ relaxations in PU-2 and PU-5.

segmental relaxation and dynamic glass transition behavior in this system. The high conductivity of the PU-urea samples originates from PEO and, in previous studies on PU nanocomposites based on the much less conductive poly(tetramethylene glycol) (PTMG) as soft segment, the $\alpha$-relaxation was clearly observed in the $\varepsilon^{\prime \prime}$ spectra. ${ }^{43}$ Analysis based on the frequency derivative of $\varepsilon^{\prime}$, which is known to result in curves similar to $\varepsilon^{\prime \prime}$ without the contribution of dc-conductivity, ${ }^{44} \mathrm{did}$ not reveal the $\alpha$-relaxation. This stresses the fact that the phenomena that hide the peaks of $\varepsilon^{\prime \prime}$ are not related to dcconductivity but rather to some Maxwell-Wagner-Sillars (MWS) polarization, ${ }^{27,40}$ or conductivity relaxation. ${ }^{24,45}$ For this reason, we followed another analysis approach. The isothermal data were re-plotted into the isochronal diagrams, which are

Table 3 Activation energy and $f_{0}$ values for $\beta$ and $\gamma$ relaxations obtained from Arrhenius plot

\begin{tabular}{|c|c|c|c|c|c|}
\hline$\gamma$ & & & $\beta$ & & \\
\hline$\underline{E_{\text {act }}}$ & & & $E_{\text {act }}$ & & \\
\hline$(\mathrm{eV})$ & $\left(\mathrm{kJ} \mathrm{mol}^{-1}\right)$ & $\log \left(f_{0}\right)$ & $(\mathrm{eV})$ & $\left(\mathrm{kJ} \mathrm{mol}^{-1}\right)$ & $\log \left(f_{0}\right)$ \\
\hline 0.38 & 36.7 & 14.4 & 0.67 & 64.7 & 18.1 \\
\hline
\end{tabular}

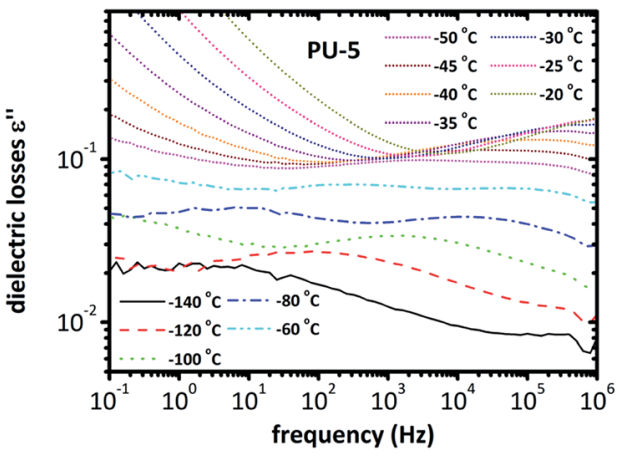

Fig. 6 Spectra of dielectric losses for PU-5 as a function of frequency, in the temperature range of $-140{ }^{\circ} \mathrm{C}$ to $-20^{\circ} \mathrm{C}$.

provided in Fig. 7 and Fig. 8, where the change in dielectric losses as a function of temperature are plotted at constant frequency of $1 \mathrm{MHz}$, where the peak is clearly apparent and the conductivity effects are minor. Positions of $\alpha$-relaxation peaks, which are related to the glass transition temperatures of the materials, are provided in Fig. 7. The $\alpha$-relaxation seems slightly slower for PU-5 compared to PU-2 and PU-8, which have similar time scales.

Isochronal diagrams of dielectric losses versus temperature for PU-5 and PU-8 are provided in Fig. 8. For frequencies higher than $0.5 \mathrm{kHz}$, two peaks are observed for PU-5 in Fig. 8a, one at low temperature, which is a superposition of $\beta$ and $\gamma$ relaxations, and the other at temperatures higher than $-50{ }^{\circ} \mathrm{C}$ with peak values of $\varepsilon^{\prime \prime}$ in the order of $10^{-1}$, due to $\alpha$-relaxation. Both peaks move to higher temperatures with increasing frequency, as expected for the thermally activated molecular polarizations. Although it is not visible in the isothermal plots, the temperature range and the height of the peak in the isochronal diagrams validate our assumption. To provide a better comparison, $\alpha$-relaxation peak was plotted for PU-8 in Fig. $8 \mathrm{~b}$. The peak was first observed above $0.5 \mathrm{kHz}$ around $-50{ }^{\circ} \mathrm{C}$ as a rather weak one and then shifted to the higher temperatures and became much more apparent as the frequency increased.

To provide a better overview, we provide the Arrhenius plot in Fig. 9, for all three samples. As can be seen in Fig. 9, these

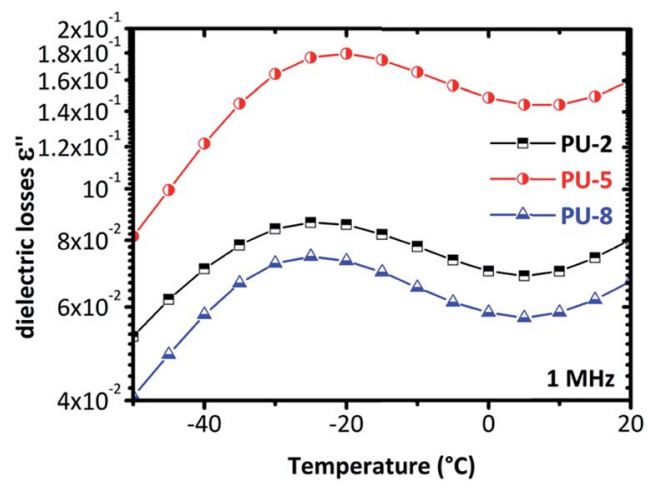

Fig. 7 The isochronal diagrams for PUUs at a frequency of $1 \mathrm{MHz}$. The peak of the PU-5 is slightly shifted to higher temperatures compared to the other two copolymers (PU-2 and PU-8). 
a.

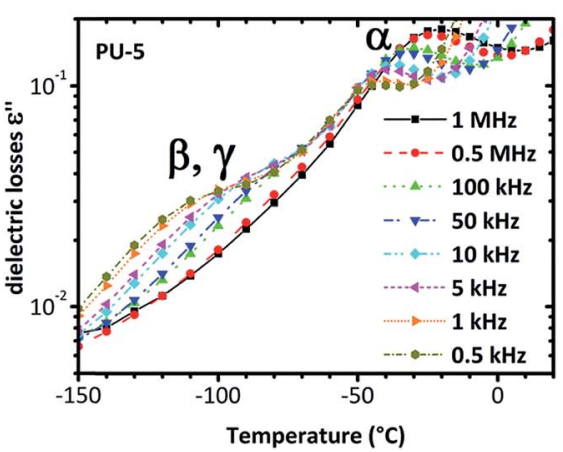

b.

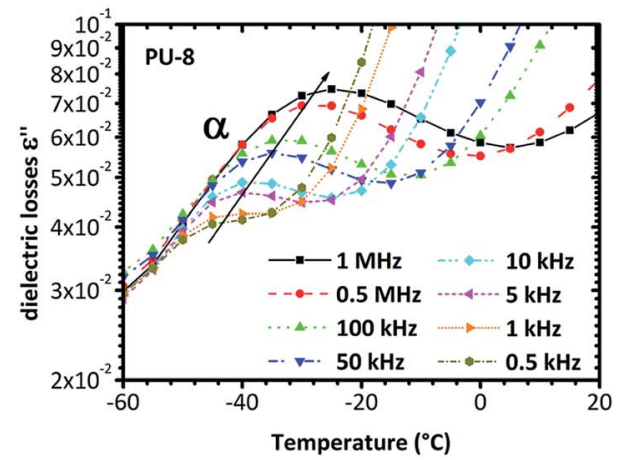

Fig. 8 (a) Isochronal diagram of dielectric losses versus temperature for PU-5. Two peaks are apparent, one ca. $-120^{\circ} \mathrm{C}$ and one ca. $-25^{\circ} \mathrm{C}$, at $1 \mathrm{MHz}$. The one at low temperatures is the sum of $\beta$ and $\gamma$ relaxations whereas the one at high temperatures is attributed to $\alpha$-relaxation or dynamic glass transition. (b) Isochronal diagram of dielectric losses versus temperature in the temperature range of $\alpha$-relaxation for the PU-8. The experimental points are noted as dots and are connected with lines. Peak moves to higher temperatures with frequency.

curves are not linear (as it was for $\beta$ and $\gamma$ relaxations given in Fig. 5) but concave. This is a typical feature of the segmental dynamics and $\alpha$-relaxation ${ }^{38}$ and confirms our assumptions about the origin of the peak. By looking at the Arrhenius plot for the $\alpha$-relaxation (Fig. 9), we can see that the time scale of the relaxation for PU-2 and PU-8 is the same (at least at high temperatures) but it is slightly slower in the case of PU-5. In addition, the curves of PU- 2 and PU- 8 have different shape that basically indicates different cooperativity. Furthermore, the $\alpha$-relaxation follows a VTF temperature dependence. The equivalent time of the relaxation for the DSC is $\tau=100 \mathrm{~s}$, so by translating this into frequency in the Arrhenius plot, we can predict the glass transition temperature for each sample. By fitting the VTF model to the points of the Arrhenius plot, here we obtained some interesting results. The fitting parameter of VTF equation for the $\alpha$-relaxation and the predicted glass transition temperature were listed in Table 4.

The Vogel temperature $\left(T_{0}\right)$ and the predicted glass transition temperature is increasing with increasing soft segment molecular weight as does the $T_{\mathrm{g}}$ determined from calorimetric measurements. This mainly suggests that the effect of

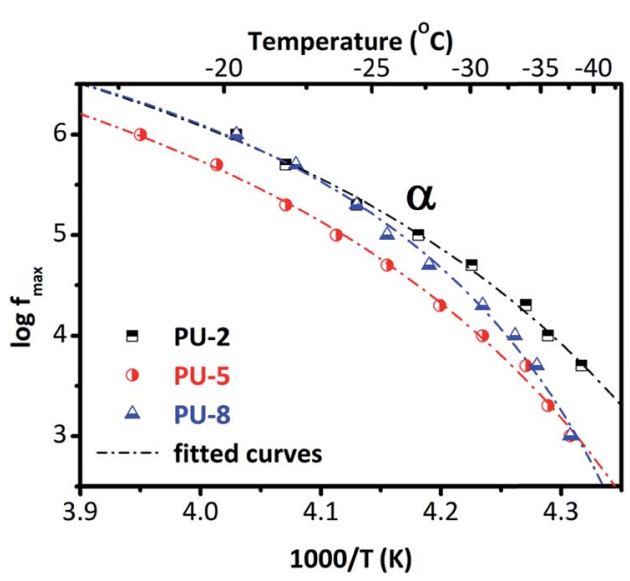

Fig. 9 Arrhenius plot for $\alpha$-relaxation for PU-2, PU-5 and PU-8. increasing degree of crystallinity dominates the behavior. Interestingly, $f_{0}$ (frequency of lattice vibrations), $B$ (apparent activation energy) and $D$ (fragility), do not follow a systematic change with the soft segment molecular weight. The highest and lowest quantitative values of these parameters were obtained for PU-5 and PU-8, respectively. Values for PU-2 were in between the other two samples. It should be noted that there is an additional uncertainty in the analysis: a single $\alpha$-relaxation was fitted to the data, although there some evidences about the existence of a slower and weaker contribution to the $\alpha$-relaxation in polyurethanes, arising from soft segments in close proximity to hard segments. ${ }^{27}$ However, this is not the case in this system. Furthermore, the behavior of $\alpha$-relaxation in PU-8 is rather interesting. As it can be seen in the Arrhenius plot (Fig. 9), it is basically similar to the one in PU-5 at low temperatures, and to the one in PU-2 at high temperatures.

3.1.3. Conductivity relaxation and interfacial MaxwellWagner-Sillars polarization. It is well-known that the conductivity in polymers is mainly associated with translation of ions whereas it is not related to the free electrons. In general, the conductivity is implemented through the diffusion of ions in the glassy matrix at temperatures below glass transition. Above glass transition, the movement of ions towards hopping over a barrier, leading to higher absolute values of conductivity. On this basis, the decrease of conductivity is usually associated with decrease in the polymer dynamics. In semi-crystalline polymers, the crystallinity restricts the movement of polymer chains, which have an impact on the overall conductivity. ${ }^{46}$

On the other hand, differences in dielectric permittivity or conductivity of the phases lead to interfacial polarization, which

Table 4 Fitting parameter of VTF equation for the $\alpha$-relaxation together with predicted and DSC glass transition temperatures

\begin{tabular}{llllllll}
\hline Sample & $\log \left(f_{\mathrm{o}}\right)$ & $B(\mathrm{~K})$ & $T_{0}(\mathrm{~K})$ & $T_{\mathrm{g}}(\mathrm{K})$ & $T_{\mathrm{g}}\left({ }^{\circ} \mathrm{C}\right)$ & ${ }^{\mathrm{DSC}} T_{\mathrm{g}}\left({ }^{\circ} \mathrm{C}\right)$ & $D$ \\
\hline PU-2 & 9.3 & 324 & 207 & 218 & -55 & -57.8 & 1.6 \\
PU-5 & 9.4 & 360 & 208 & 220 & -53 & -51.9 & 1.7 \\
PU-8 & 8.6 & 185 & 217 & 224 & -49 & -48.4 & 0.9
\end{tabular}


can be described as the accumulation of space charges at the interfaces between the various phases, in heterogeneous materials like phase separated polymers. The accumulating charges behave like a dipole giving rise to the formation of a peak in the dielectric loss spectra, which is generally referred as Maxwell-Wagner-Sillars (MWS) polarization. ${ }^{7,12}$ More specifically, this interfacial polarization is originated from mobility and accumulation of charges at the interfaces of soft and hard domains of polyurethanes due to the differences between their respective dielectric constants and conductivities, which also affect the frequency and intensity of the MWS process. ${ }^{7}$ Therefore, the interfacial MWS polarization can be examined for a better understanding of micro-phase separation in polyurethanes.

As mentioned earlier, the dielectric analysis, particularly following the $\alpha$-relaxation, was quite challenging in this system due to high conductivity of PEO. For this reason, we tried to apply a better approach to the system by comparing diagrams of different quantities obtained using different formalisms, such as the $\left(\varepsilon^{\prime}\right)$ and $\left(\varepsilon^{\prime \prime}\right)$, their ratio $(\tan \delta)$, the conductivity $\left(\sigma_{\mathrm{ac}}\right)$, the imaginary part of the electric modulus $\left(M^{\prime \prime}\right)$ and the frequency derivative of $\left(\varepsilon^{\prime}\right)$. From these plots, it is possible to correlate different curves to various phenomena, such as interfacial Maxwell-Wagner-Sillars (MWS) polarization, conductivity relaxation, etc. ${ }^{40}$ The multi-plots of dielectric the losses $\left(\varepsilon^{\prime \prime}\right)$, the electric modulus $\left(M^{\prime \prime}\right)$ and the conductivity, $\left(\sigma_{\mathrm{ac}}\right)$ for PU-2 and PU-5 are given in Fig. 10a and b, respectively. In the spectrum of electric modulus $\left(M^{\prime \prime}\right)$, the peak at low frequencies is related to a conductivity relaxation, i.e. the transition from conductivity DC (which is frequency independent) to conductivity AC (which increases linearly with frequency in a log-log plot). By comparing the spectra of electric modulus and conductivity, it should be noted that the peak of $\left(M^{\prime \prime}\right)$ is slightly higher than the "knee" of the conductivity. ${ }^{24}$

For this system, at temperatures higher than $-20{ }^{\circ} \mathrm{C}$, interfacial MWS polarization dominates the spectrum of $\left(\varepsilon^{\prime \prime}\right)$. To provide a clear overview on the conductivity relaxation and interfacial MWS polarization, the Arrhenius plot was reconstructed by using the Havriliak-Negami model for the peaks of $\left(M^{\prime \prime}\right)$ for all three samples in Fig. 11.
As can clearly be seen in Fig. 11, all the samples under investigation (PU-2, PU-5 and PU-8) display quite similar behaviors, and thus, similar activation energies in terms of interfacial MWS polarization. Although these results indicate that the microphase separation is not significantly affected by the soft segment molecular weight, it should be noted that no safe conclusions can be extracted here due to the complexity of the system and the presence of many phases with different conductivity. For this reason, the interfacial MWS polarization will not be further discussed.

On the other hand, significant differences were observed in the conductivity relaxation, which is much faster for PU-2 (Fig. 11). This is mainly related to the amorphous/crystalline nature of the samples. As the conductivity decreases with increasing crystallinity, which is due to the restrained mobility arisen from the presence of the crystalline regions, the conductivity relaxation is expected to be slower for the samples with high crystallinity values. Since PU-2 copolymer is mainly amorphous, there is no notable restriction on the mobility, particularly in comparison to other two crystalline materials under investigation (PU-5 and PU-8), its conductivity relaxation becomes faster than those of PU-5 and PU-8. Besides, the shape of the Arrhenius curves for the interfacial MWS polarization and conductivity relaxation is similar to each other (Fig. 11). Parallel

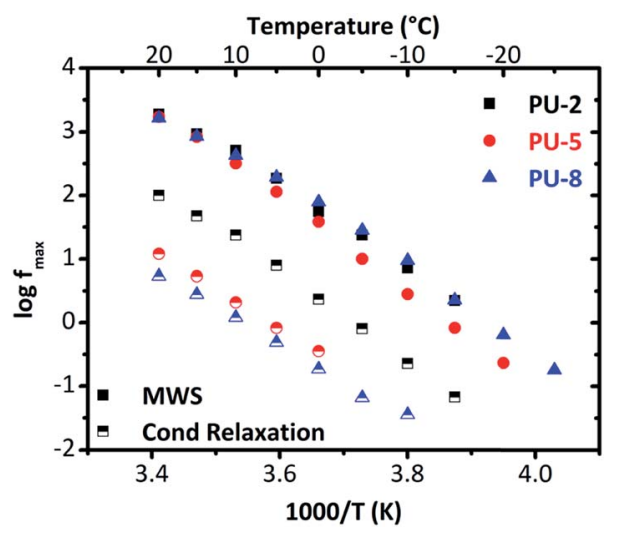

Fig. 11 Arrhenius plot derived from the electric modulus diagrams for the MWS polarization and the conductivity relaxation. a.

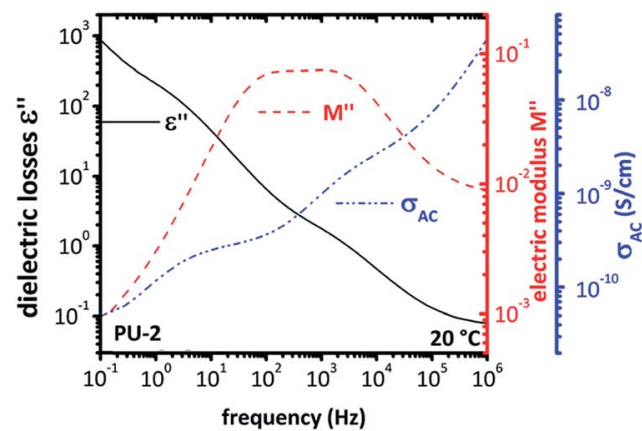

b.

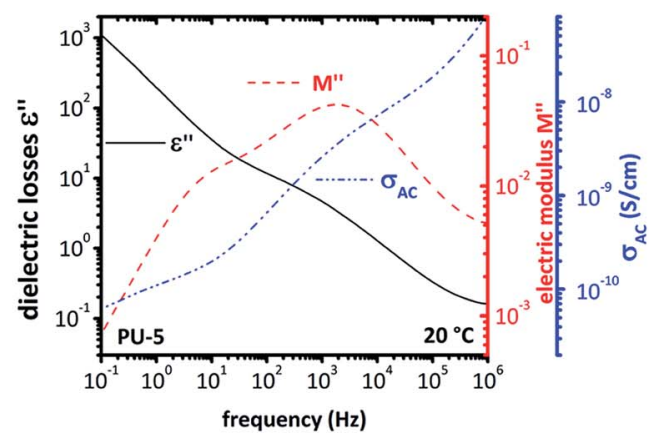

Fig. 10 Multi-plots for (a) PU-2 and (b) PU-5 in order to compare the dielectric losses $\left(\varepsilon^{\prime \prime}\right)$, the electric modulus $\left(M^{\prime \prime}\right)$ and the conductivity $\left(\sigma_{\text {ac }}\right)$. 
traces of these two processes for each sample mainly imply similar activation energies. In addition, both of the processes, i.e., MWS polarization and conductivity relaxation, follow VTF behavior, which is typical for the conductivity relaxation above glass transition temperature, basically indicating cooperativity in the system.

\section{Conclusions}

The effect of soft segment molecular weight on the molecular mobility and segmental dynamics of a series of PEO-based polyurethaneurea copolymers with identical hard segment contents ( $30 \%$ by weight) were investigated using differential scanning calorimetry and dielectric relaxation spectroscopy. DSC analyses showed that PEO SS glass transition temperature increased with the soft segment molecular weight. This was supported by the results obtained from DRS analyses. Furthermore, as widely observed in polyurethanes, these copolymers also display rich dielectric relaxation behavior, where five different relaxations were observed in dielectric analyses of all copolymers. These were local glassy state motions ( $\gamma$ and $\beta$ ), segmental motion of the soft phase $(\alpha)$, conductivity relaxation (CR) and interfacial Maxwell-Wagner-Sillars (MWS) polarization. Local relaxations follow Arrhenius behavior and their time scale is not affected by the soft segment molecular weight. Interestingly enough, the $\alpha$-relaxation was not visible in the spectra of dielectric losses versus frequency, but became apparent after re-plotting the data into isochronal diagrams. It follows VTF behavior and interestingly is slower for the copolymer based on PEO-4600 (PU-5) when compared with those based on PEO-2000 (PU-2) and PEO-8000 (PU-8). The conductivity relaxation and the interfacial MWS polarization also follow VTF behavior and have quite similar slope basically indicating cooperativity and similar activation energies, respectively. Very interestingly, the interfacial MWS polarization is not affected by the soft segment molecular weight indicating that microphase separation is not significantly affected by the PEO molecular weight for this system, which is unexpected. Due to the highly complex structures of the samples having many phases with different conductivity, we should note that no safe conclusions can be extracted for the microphase separation in this system based on the interfacial MWS polarization. However, significant differences were observed in the conductivity relaxation, which is much faster for PU-2 copolymer when compared with PU-5 and PU-8. This is explained by an increase in the degree of crystallinity of the PEO matrix with an increase in the PEO molecular weight in these copolymers, as demonstrated by XRD and DSC studies.

\section{Conflicts of interest}

The authors declare no conflict of interest.

\section{Acknowledgements}

We gratefully acknowledge Scientific and Technical Research Council of Turkey (TUBITAK) for the use of materials which were synthesized within the content of the project funded under the contact number 109M073.

\section{References}

1 A. Noshay and J. E. McGrath, Block Copolymers: Overview and Critical Survey, Elsevier, 2013.

2 M. Szycher, Szycher's Handbook of Polyurethanes, Taylor \& Francis, 1st edn, 1999.

3 P. Król, Prog. Mater. Sci., 2007, 52, 915-1015.

4 I. Yilgör, E. Yilgör and G. L. Wilkes, Polymer, 2015, 58, A1A36.

5 A. M. Castagna, D. Fragiadakis, H. Lee, T. Choi and J. Runt, Macromolecules, 2011, 44, 7831-7836.

6 A. M. Castagna, A. Pangon, T. Choi, G. P. Dillon and J. Runt, Macromolecules, 2012, 45, 8438-8444.

7 D. Fragiadakis and J. Runt, Macromolecules, 2013, 46, 41844190.

8 K. N. Raftopoulos, B. Janowski, L. Apekis, K. Pielichowski and P. Pissis, Eur. Polym. J., 2011, 47, 2120-2133.

9 K. N. Raftopoulos, S. Koutsoumpis, M. Jancia, J. P. Lewicki, K. Kyriakos, H. E. Mason, S. J. Harley, E. Hebda, C. M. Papadakis, K. Pielichowski and P. Pissis, Macromolecules, 2015, 48, 1429-1441.

10 C. Schick, Eur. Phys. J.: Spec. Top., 2010, 189, 3-36.

11 B. Wunderlich, Thermal Analysis of Polymeric Materials, Springer, Berlin Heidelberg, 2005.

12 F. Kremer and A. Schönhals, Broadband Dielectric Spectroscopy, Springer, Berlin Heidelberg, 2002.

13 E. Akiyama, Y. Nagase, N. Koide and K. Araki, Phys. Chem. Chem. Phys., 1999, 1, 2319-2326.

14 Y. W. Lian, K. S. Zhao and L. K. Yang, Phys. Chem. Chem. Phys., 2010, 12, 6732-6741.

15 C. Colosi, M. Costantini, A. Barbetta, C. Cametti and M. Dentini, Phys. Chem. Chem. Phys., 2013, 15, 20153-20160.

16 M. Sharma, G. Madras and S. Bose, Phys. Chem. Chem. Phys., 2014, 16, 23421-23430.

17 J. Li and K. Zhao, Phys. Chem. Chem. Phys., 2015, 17, 41754183.

18 T. Hirata, H. Matsuno, D. Kawaguchi, M. Inutsuka, T. Hirai, M. Tanaka and K. Tanaka, Phys. Chem. Chem. Phys., 2017, 19, 1389-1394.

19 S. Kitajima, F. Bertasi, K. Vezzù, E. Negro, Y. Tominaga and V. Di Noto, Phys. Chem. Chem. Phys., 2013, 15, 16626-16633. 20 A. Campanella, A. Brás, K. N. Raftopoulos, C. M. Papadakis, O. Vassiliadou, A. Kyritsis, M. S. Appavou, P. MüllerBuschbaum and H. Frielinghaus, Polymer, 2016, 96, 70-80.

21 A. G. Charnetskaya, G. Polizos, V. I. Shtompel, E. G. Privalko, Y. Y. Kercha and P. Pissis, Eur. Polym. J., 2003, 39, 2167-2174.

22 T. Choi, D. Fragiadakis, C. M. Roland and J. Runt, Macromolecules, 2012, 45, 3581-3589.

23 D. Fragiadakis, S. Dou, R. H. Colby and J. Runt, Macromolecules, 2008, 41, 5723-5728.

24 G. Georgoussis, A. Kanapitsas, P. Pissis, Y. V. Savelyev, V. Y. Veselov and E. G. Privalko, Eur. Polym. J., 2000, 36, 1113-1126. 
25 A. Kanapitsas and P. Pissis, Eur. Polym. J., 2000, 36, 12411250.

26 A. Kanapitsas, P. Pissis and A. Garcia Estrella, Eur. Polym. J., 1999, 35, 923-937.

27 K. N. Raftopoulos, B. Janowski, L. Apekis, P. Pissis and K. Pielichowski, Polymer, 2013, 54, 2745-2754.

28 K. N. Raftopoulos, C. Pandis, L. Apekis, P. Pissis, B. Janowski, K. Pielichowski and J. Jaczewska, Polymer, 2010, 51, 709-718.

29 K. Chrissopoulou, K. S. Andrikopoulos, S. Fotiadou, S. Bollas, C. Karageorgaki, D. Christofilos, G. A. Voyiatzis and S. H. Anastasiadis, Macromolecules, 2011, 44, 9710-9722.

30 A. C. French, A. L. Thompson and B. G. Davis, Angew. Chem., Int. Ed., 2009, 121, 1274-1278.

31 S. A. Kim and L. A. Archer, Macromolecules, 2014, 47, 687694.

32 H. Matsuura and K. Fukuhara, J. Polym. Sci., Part B: Polym. Phys., 1986, 24, 1383-1400.

33 J. Maxfield and I. W. Shepherd, Polymer, 1975, 16, 505-509.

34 W. Qiu, M. Pyda, E. Nowak-Pyda, A. Habenschuss and B. Wunderlich, Macromolecules, 2005, 38, 8454-8467.

35 T. Uemura, N. Yanai, S. Watanabe, H. Tanaka, R. Numaguchi, M. T. Miyahara, Y. Ohta, M. Nagaoka and S. Kitagawa, Nat. Commun., 2010, 1, 83.
36 O. Malay, O. Oguz, C. Kosak, E. Yilgor, I. Yilgor and Y. Z. Menceloglu, Polymer, 2013, 54, 5310-5320.

37 S. Havriliak and S. Negami, Polymer, 1967, 8, 161-210.

38 E. J. Donth, The Glass Transition: Relaxation Dynamics in Liquids and Disordered Materials, Springer, 2001.

39 P. Klonos, A. Panagopoulou, L. Bokobza, A. Kyritsis, V. Peoglos and P. Pissis, Polymer, 2010, 51, 5490-5499.

40 A. Kyritsis, G. Vikelis, P. Maroulas, P. Pissis, B. Milosheva, R. Kotsilkova, A. Toplijska, C. Silvestre and D. Duraccio, J. Appl. Polym. Sci., 2011, 121, 3613-3627.

41 O.-H. Kwon, V. Ortalan and A. H. Zewail, Proc. Natl. Acad. Sci. U. S. A., 2011, 108, 6026-6031.

42 F. E. Bailey and J. V. Koleske, Poly(ethylene oxide), Academic Press, 1976.

43 S. Koutsoumpis, K. N. Raftopoulos, M. Jancia, J. Pagacz, E. Hebda, C. M. Papadakis, K. Pielichowski and P. Pissis, Macromolecules, 2016, 49, 6507-6517.

44 J. van Turnhout and M. Wübbenhorst, J. Non-Cryst. Solids, 2002, 305, 50-58.

45 E. Neagu, P. Pissis, L. Apekis and J. G. Ribelles, J. Phys. D: Appl. Phys., 1997, 30, 1551.

46 S. Kripotou, P. Pissis, V. A. Bershtein, P. Sysel and R. Hobzova, Polymer, 2003, 44, 2781-2791. 